\title{
Expression levels of transcription factors c-Fos and c-Jun and transmembrane protein HAb18G/CD147 in urothelial carcinoma of the bladder
}

\author{
MUREN HUHE* ${ }^{*}$ SHUANGSHUANG LIU*, YANG ZHANG*, ZHENG ZHANG and ZHINAN CHEN \\ Cell Engineering Research Center and Department of Cell Biology, State Key Laboratory of Cancer Biology, \\ Fourth Military Medical University, Xi'an, Shaanxi 710032, P.R. China
}

Received December 27, 2015; Accepted February 6, 2017

DOI: $10.3892 / \mathrm{mmr} .2017 .6411$

\begin{abstract}
The aim of the present study was to investigate the prognostic significance of the expression of transcription factors, c-Fos, c-Jun and transmembrane protein CD147, in urothelial carcinoma of the bladder (UCB). The current study investigated the clinical significance of these factors in the development, progression and survival analysis of UCB. Immunohistochemistry was employed to analyze c-Fos, c-Jun and CD147 expression in 41 UCB cases and 34 non-cancerous human bladder tissues. These results were scored in a semi-quantitative manner based on the intensity and percentage of tumor cells that presented immunoreactivity. Protein levels of CD147, c-Fos and c-Jun expression were upregulated in 22 (53.7\%), 10 (24.4\%) and 9 (22.0\%) UCB cases, respectively. High levels of c-Jun correlated with the AJCC cancer staging manual (7th edition; $\mathrm{P}=0.038$ ). Univariate analysis revealed that upregulated CD147 $(\mathrm{P}=0.038)$ or $\mathrm{c}-\mathrm{Jun}(\mathrm{P}=0.008)$ was associated with poor overall survival (OS), respectively. Further analysis revealed that either CD147-c-Fos-c-Jun co-expression $(\mathrm{P}=0.004)$, or CD147-c-Jun co-expression $(\mathrm{P}=0.037)$ and $c-F o s-c-J u n$ co-expression $(\mathrm{P}<0.001)$ were associated with poor OS. Multivariate analysis suggested that either upregulation of CD147, c-Jun or c-Fos were independent risk indicators for death in UCB patients. Increased expression of c-Jun or CD147, as well as co-expression of CD147-c-Jun, c-Jun-c-Fos or CD147-c-Jun-c-Fos has prognostic significance for UCB patients. Therefore, high CD147 and c-Jun expression
\end{abstract}

Correspondence to: Professors Zhinan Chen or Zheng Zhang, Cell Engineering Research Center and Department of Cell Biology, State Key Laboratory of Cancer Biology, Fourth Military Medical University, 169 Changle Road, Xi'an, Shaanxi 710032, P.R. China

E-mail: zhinanchen@fmmu.edu.cn

E-mail: zhangzzy@aliyun.com

${ }^{*}$ Contributed equally

Key words: CD147, c-Jun, c-Fos, urothelial carcinoma of the bladder may serve roles in tumor progression and may be diagnostic and therapeutic targets in UCB whether alone or in combination.

\section{Introduction}

Bladder cancer is the second most common urologic malignancy amongst males (1). In 2015 in the United States, it was estimated that there were 74,000 new cases of bladder cancer and 16,000 deaths (2). Transitional cell carcinoma (TCC) or urothelial carcinoma of the bladder (UCB) account for $90 \%$ of all bladder cancer cases, and are divided in 2 variants: non-muscle invasive or muscle invasive. The non-muscle invasive form has a high rate of recurrence $(50 \%)$, and it progresses to muscle invasive form in $\sim 11 \%$ of the cases (3). Despite advances in multi-treatment approaches, including surgery, radiotherapy and chemotherapy, the clinical outcomes of bladder cancer patients remain unsatisfactory $(4,5)$. This is due to considerable tumor variability and heterogeneity, even within the same pathological stage. Predicting a prognosis by examining the clinicopathological characteristics remains difficult. Therefore, identification of novel prognostic factors with high sensitivity and specificity is important to evaluate the prognosis of UCB and individualize treatment strategies.

CD147 is a member of the immunoglobulin superfamily. It is widely expressed in human tumors and plays a central role in the progression of many cancers by stimulating the secretion of matrix metalloproteinases (MMPs) and cytokines (6). CD147 regulates cell proliferation, apoptosis, metastasis, differentiation and chemical drug resistance (6). In addition, recent studies have demonstrated that the level of CD147 is associated with the prognosis of patients in many cancers (6). Furthermore, CD147 is recognized as an effective therapeutic target for hepatocellular carcinoma (HCC) $(7,8)$ and additional cancers $(9,10)$, and exciting clinical progress has been made in HCC treatment with CD147-directed monoclonal antibodies (11).

The activator protein-1 transcription factor, AP-1, is principally composed of homodimers of the Jun family (c-Jun, JunB and JunD), heterodimers of the members of Fos (c-Fos, FosB, Fra-1 and Fra-2) and Jun, or cAMP response 
element-binding protein/activating transcription factor family members (12). Homo- or hetero- forms of the AP-1 complex can activate target gene expression by binding to 12-O-Tetradecanoylphorbol-13-acetate-(or TPA)-responsive elements, which are also known as AP-1 sites (consensus sequence, 5'-TGAG/CTCA-3'), in promoter or enhancer regions (12). Evidence has demonstrated that AP-1 serves an important role in inflammation, cellular migration, metastasis and cell proliferation, apoptosis and transformation (13). More importantly, several studies have revealed that the AP-1 complex is involved in carcinogenesis of various tumor types, such as ovarian cancer, breast cancer and skin cancer. For example, c-Fos has been identified as independent predictor of decreased survival in breast cancer (14). High levels of c-Fos expression are associated with high-grade lesions and poor prognosis in osteosarcoma and endometrial carcinoma $(15,16)$. High levels of c-Jun protein have been identified in $31 \%$ of primary and metastatic lung tumors (17). In breast cancer, activated c-Jun is expressed predominantly at the invasive front of breast cancer and is associated with proliferation and angiogenesis (18). However, there has been little research into the levels of c-Jun and c-Fos in patients with urothelial carcinoma of bladder tissues.

In the present study, the prognostic significance of c-Fos, c-Jun and CD147 expression was examined by performing immunohistochemistry with a tissue microarray of samples from 41 patients with UCB. In addition, the correlation between c-Fos, c-Jun and CD147 expression in UCB was investigated to supply more reliable judgments of the prognosis of UCB patients.

\section{Materials and methods}

Patients and tissues. A total of 41 bladder cancer tissues with histologically confirmed UCB were obtained from the Shanghai Outdo Biotech Co., Ltd. (Shanghai, China) from May 2007 to November 2011, and 34 para-cancer normal tissues, which were $5 \mathrm{~cm}$ far away from the edge of the tumor, were randomly selected as controls. The study was approved by the Ethics Committee of the Fourth Military Medical University (Xi'an, China). Following transurethral resection and partial or radical cystectomy therapy, all patients were available for follow-up data and provided written and informed consent. Clinical pathological information was obtained from patients' operative and pathological reports, and included: Sex, age, recurrence, metastasis, invasion tumor size and growth pattern, WHO grade, TNM stage, AJCC stage and survival time.

The study population comprised 35 men (85.4\%) and 6 women $(14.6 \%)$, with a median age at diagnosis of 71 years (range, 44-85 years). The tumor growth type was papillary in 11 patients $(26.8 \%)$ and nonpapillary in 30 patients $(73.2 \%)$; low-grade tumors were observed in 3 patients $(7.3 \%)$ and high-grade tumors were observed in 38 patients $(92.7 \%)$. Furthermore, 6 patients $(14.6 \%)$ had low-stage (Tis) tumors and 35 patients $(85.4 \%)$ had high-stage tumors (T1, 6; T2, 10; T3, 15; T4a, 4; Table I). There was no recurrence or distant metastasis recorded in all cases. Among these 41 patients, $18(43.9 \%)$ did not survive. The median follow-up period was 35 months (data not shown).
Immunohistochemical (IHC) staining and evaluation. The expression of c-Jun, c-Fos and CD147 was detected by IHC staining. Briefly, $5 \mu \mathrm{m}$ sections were cut from formalin-fixed, paraffin-embedded tissues. Following deparaffinization and dehydration, sections were heated in an autoclave $\left(\sim 120^{\circ} \mathrm{C}\right.$, $200 \mathrm{kPa}, 2$ to $6 \mathrm{~min}$,) in $10 \mathrm{mM}$ citrate buffer ( $\mathrm{pH} \mathrm{6.0)} \mathrm{for}$ antigen retrieval, and treated with $3 \% \mathrm{H}_{2} \mathrm{O}_{2}$ for 20 min to block the peroxidase. Sections were blocked in $5 \%$ goat serum in PBS, followed by overnight incubation at $4^{\circ} \mathrm{C}$ with anti-c-Jun antibody (1:100 dilution; cat. no. \#9165 Cell Signaling Technology, Inc., Danvers, MA, USA), anti-c-Fos antibody (1:500 dilution; cat. no. \#2251; Cell Signaling Technology, Inc.) andanti-CD147 antibody (1:167 dilution; cat. no. \#201508; Cell Engineering Research Center, Fourth Military Medical University, Xi'an, China). The signal was detected with streptavidin-peroxidase staining kits (Thermo Fisher Scientific, Inc., Waltham, MA, USA) and DAB substrate (ZSGB-BIO., Beijing, China). All sections were then counterstained with Mayer's hematoxylin $(0.1 \%)$ for 5 to $8 \mathrm{~min}$ at room temperature (cat. no \#715012; Baso Diagnostics, Inc., Zhuhai, Guangdong, China) and then mounted.

Images were taken with an inverted microscope (CKX41; Olympus Corporation, Tokyo, Japan) equipped with a digital camera under x100 and x400 magnification. Two pathologists evaluated the results independently, with no knowledge of clinicopathological features.

The expression of c-Jun, c-Fos and CD147 were scored in a semi-quantitative manner according both the extent and the intensity of the staining: IHC negative, absent; $\mathrm{IHC}+$, faint or weak intensity in $>10 \%$ of cancer cells; $\mathrm{IHC}++$, moderate intensity in $>10 \%$ of cancer cells; and $\mathrm{IHC}+++$, strong intensity in $>10 \%$ of cancer cells.

Statistical analysis. All statistical analyses were performed using SPSS software (version, 17.0; SPSS Inc., Chicago, IL, USA). The relationship between the level of three proteins (c-Fos, c-Jun and CD147) and clinicopathological features was analyzed with chi-squared statistical test (linear-by-linear association or the Fisher exact test) or Spearman correlation analysis, while overall survival (OS) analysis was carried out using the Kaplan-Meier method and compared by the Log-rank test and the Cox proportional hazards regression model served to identify relevant prognostic factors. All P-values were two sided and $\mathrm{P}<0.05$ was considered to indicate a statistically significant difference.

\section{Results}

The expression characteristicss of c-Jun, c-Fos and CD147 in UCB and normal tissues. A total of 9 of 41 (22.0\%) UCB tissues and 1 of $34(8.8 \%)$ para-cancer tissues presented positive staining of c-Jun, respectively. Tumor staining of c-Jun expression was observed in the nucleus staining pattern (Fig. 1), however, only negative or weak immunoreactivity of c-Jun was detected in para-cancer tissues, which indicated that positive c-Jun was more commonly expressed in human UCB than para-cancer tissues $(\mathrm{P}=0.017$; Fig. 1$)$.

Following IHC in UCB tissues, 10 of 41 cases $(24.4 \%)$ presented c-Fos staining, which was strong in 1 case $(2+$ or higher, $2.4 \%)$, and weak in 9 cases $(1+, 22.0 \%)$. The other 
Table I. CD147, c-Fos, and c-Jun expression in 41 patients with urothelial carcinoma of the bladder.

\begin{tabular}{|c|c|c|c|c|c|}
\hline \multirow[b]{2}{*}{ Factor } & \multicolumn{4}{|c|}{ CD147 (\%) } & \multirow[b]{2}{*}{ P-value } \\
\hline & $\begin{array}{c}+++ \\
(\mathrm{n}=3)(\%)\end{array}$ & $\begin{array}{c}++ \\
(\mathrm{n}=6)(\%)\end{array}$ & $\begin{array}{c}+ \\
(\mathrm{n}=13)(\%)\end{array}$ & $\begin{array}{c}- \\
(\mathrm{n}=19)(\%)\end{array}$ & \\
\hline \multicolumn{6}{|l|}{ Age (y) } \\
\hline$>71(\mathrm{n}=20)$ & $1(5.0)$ & $2(10.0)$ & $7(35.0)$ & $10(50.0)$ & 0.393 \\
\hline$\leq 71(n=21)$ & $2(9.5)$ & $4(19.0)$ & $6(28.6)$ & $9(42.9)$ & \\
\hline \multicolumn{6}{|l|}{ Sex } \\
\hline Male $(n=35)$ & $3(8.6)$ & $6(17.1)$ & $11(31.4)$ & $15(42.9)$ & 0.165 \\
\hline Female $(n=6)$ & $0(0)$ & $0(0)$ & $2(33.3)$ & $4(66.7)$ & \\
\hline \multicolumn{6}{|l|}{ Growth pattern } \\
\hline Papillary $(\mathrm{n}=11)$ & $0(0)$ & $2(18.2)$ & $5(45.5)$ & $4(36.4)$ & 0.964 \\
\hline Nonpapillary $(\mathrm{n}=30)$ & $3(10.0)$ & $4(13.3)$ & $8(26.7)$ & $15(50.0)$ & \\
\hline \multicolumn{6}{|l|}{ WHO grade } \\
\hline Low $(\mathrm{n}=3)$ & $0(0)$ & $1(33.3)$ & $2(66.7)$ & $0(0)$ & 0.338 \\
\hline $\operatorname{High}(\mathrm{n}=38)$ & $3(7.9)$ & $5(13.2)$ & $11(28.9)$ & $19(50.0)$ & \\
\hline \multicolumn{6}{|l|}{ Lymph node invasion } \\
\hline Negative $(n=37)$ & $2(5.4)$ & $6(16.2)$ & $10(27.0)$ & $19(51.4)$ & 0.136 \\
\hline Positive $(n=4)$ & $1(25.0)$ & $0(0)$ & $3(75.0)$ & $0(0)$ & \\
\hline \multicolumn{6}{|l|}{ TNM stage } \\
\hline Low (Tis) $(\mathrm{n}=6)$ & $0(0)$ & $0(0)$ & $4(66.7)$ & $2(33.3)$ & 0.649 \\
\hline $\operatorname{High}(\mathrm{T} 1-\mathrm{T} 4 \mathrm{a})(\mathrm{n}=35)$ & $3(8.6)$ & $6(17.1)$ & $9(25.7)$ & $17(48.6)$ & \\
\hline \multicolumn{6}{|l|}{ Tumor diameter } \\
\hline$>4 \mathrm{~cm}(\mathrm{n}=16)$ & $0(0)$ & $5(31.3)$ & $6(37.5)$ & $5(31.3)$ & 0.355 \\
\hline$\leq 4 \mathrm{~cm}(\mathrm{n}=25)$ & $3(12.0)$ & $1(4.0)$ & $7(28.0)$ & $14(56.0)$ & \\
\hline \multicolumn{6}{|l|}{ AJCC cancer staging } \\
\hline Tis $(\mathrm{n}=12)$ & $1(8.3)$ & $1(8.3)$ & $4(33.3)$ & $6(50.0)$ & 0.554 \\
\hline $1-2(n=11)$ & $0(0)$ & $2(18.2)$ & $4(36.4)$ & $5(45.5)$ & \\
\hline \multirow[t]{3}{*}{$3-4(n=18)$} & $2(11.1)$ & $5(27.8)$ & $3(16.7)$ & $8(44.4)$ & \\
\hline & \multicolumn{5}{|c|}{ c-Fos $(\%)$} \\
\hline & $\begin{array}{l}+++ \\
(\mathrm{n}=0)\end{array}$ & $\begin{array}{c}++ \\
(\mathrm{n}=1)(\%)\end{array}$ & $\begin{array}{c}+ \\
(\mathrm{n}=9)(\%)\end{array}$ & $\begin{array}{c}-(\mathrm{n}=31) \\
(\%)\end{array}$ & P-value \\
\hline \multicolumn{6}{|l|}{ Age $(y)$} \\
\hline$>71(n=20)$ & - & $0(0)$ & $6(30.0)$ & $14(70.0)$ & 0.693 \\
\hline$\leq 71(\mathrm{n}=21)$ & - & $1(4.8)$ & $3(14.3)$ & $17(81.0)$ & \\
\hline \multicolumn{6}{|l|}{ Sex } \\
\hline Male $(n=35)$ & - & $1(2.9)$ & $6(17.1)$ & $28(80.0)$ & 0.220 \\
\hline Female $(n=6)$ & - & $0(0)$ & $3(50.0)$ & $3(50.0)$ & \\
\hline \multicolumn{6}{|l|}{ Growth pattern } \\
\hline Papillary $(\mathrm{n}=11)$ & - & $1(9.1)$ & $1(9.1)$ & $9(81.8)$ & 0.973 \\
\hline Nonpapillary $(\mathrm{n}=30)$ & - & $0(0)$ & $8(26.7)$ & $22(73.3)$ & \\
\hline \multicolumn{6}{|l|}{ WHO grade } \\
\hline Low $(n=3)$ & - & $0(0)$ & $0(0)$ & $3(100.0)$ & 0.336 \\
\hline $\operatorname{High}(\mathrm{n}=38)$ & - & $1(2.6)$ & $9(23.7)$ & $28(73.7)$ & \\
\hline \multicolumn{6}{|l|}{ Lymph node invasion } \\
\hline Negative $(n=37)$ & - & $1(2.7)$ & $7(18.9)$ & $29(78.4)$ & 0.330 \\
\hline Positive $(n=4)$ & - & $0(0)$ & $2(50.0)$ & $2(50.0)$ & \\
\hline \multicolumn{6}{|l|}{ TNM stage } \\
\hline Low (Tis) $(n=6)$ & - & $1(16.7)$ & $0(0)$ & $5(83.3)$ & 0.731 \\
\hline $\operatorname{High}(\mathrm{T} 1-\mathrm{T} 4 \mathrm{a})(\mathrm{n}=35)$ & - & $0(0)$ & $9(25.7)$ & $26(74.3)$ & \\
\hline
\end{tabular}


Table I. Continued.

\begin{tabular}{|c|c|c|c|c|c|}
\hline \multirow[b]{2}{*}{ Factor } & \multicolumn{4}{|c|}{$\mathrm{c}-\mathrm{Fos}(\%)$} & \multirow[b]{2}{*}{ P-value } \\
\hline & $\begin{array}{l}+++ \\
(\mathrm{n}=0)\end{array}$ & $\begin{array}{c}++ \\
(\mathrm{n}=1)(\%)\end{array}$ & $\begin{array}{c}+ \\
(\mathrm{n}=9)(\%)\end{array}$ & $\begin{array}{c}-(\mathrm{n}=31) \\
(\%)\end{array}$ & \\
\hline \multicolumn{6}{|l|}{ Tumor diameter } \\
\hline$>4 \mathrm{~cm}(\mathrm{n}=16)$ & - & $1(6.3)$ & $3(18.8)$ & $12(75.0)$ & 0.651 \\
\hline$\leq 4 \mathrm{~cm}(\mathrm{n}=25)$ & - & $0(0)$ & $6(24.0)$ & $19(76.0)$ & \\
\hline \multicolumn{6}{|l|}{ AJCC cancer staging } \\
\hline Tis $(n=12)$ & - & $1(8.3)$ & $2(16.7)$ & $9(75.0)$ & 0.552 \\
\hline $1-2(n=11)$ & - & $0(0)$ & $3(27.3)$ & $8(72.7)$ & \\
\hline \multirow[t]{2}{*}{$3-4(n=18)$} & - & $0(0)$ & $4(22.2)$ & $14(77.8)$ & \\
\hline & \multicolumn{5}{|c|}{ c-Jun (\%) } \\
\hline Factor & $+++(\mathrm{n}=0)$ & $++(\mathrm{n}=0)$ & $+(\mathrm{n}=9)(\%)$ & $-(\mathrm{n}=32)(\%)$ & P-value \\
\hline \multicolumn{6}{|l|}{ Age (y) } \\
\hline$>71(\mathrm{n}=20)$ & - & - & $2(10.0)$ & $18(90.0)$ & 0.130 \\
\hline$\leq 71(\mathrm{n}=21)$ & - & - & $7(33.3)$ & $14(66.7)$ & \\
\hline \multicolumn{6}{|l|}{ Sex } \\
\hline Male (n=35) & - & - & $8(22.9)$ & $27(77.1)$ & 1.000 \\
\hline Female $(n=6)$ & - & - & $1(16.7)$ & $5(83.3)$ & \\
\hline \multicolumn{6}{|l|}{ Growth pattern } \\
\hline Papillary (n=11) & - & - & $2(18.2)$ & $9(81.8)$ & 1.000 \\
\hline Nonpapillary $(\mathrm{n}=30)$ & - & - & $7(23.3)$ & $23(76.7)$ & \\
\hline \multicolumn{6}{|l|}{ WHO grade } \\
\hline Low $(n=3)$ & - & - & $0(0)$ & $3(100)$ & 1.000 \\
\hline High $(n=38)$ & - & - & $9(23.7)$ & $29(76.3)$ & \\
\hline \multicolumn{6}{|l|}{ Lymph node invasion } \\
\hline Negative $(n=37)$ & - & - & $7(18.9)$ & $30(81.1)$ & 0.204 \\
\hline Positive $(n=4)$ & - & - & $2(50.00)$ & $2(50.0)$ & - \\
\hline \multicolumn{6}{|l|}{ TNM stage } \\
\hline Low (Tis) $(\mathrm{n}=6)$ & - & - & $2(33.3)$ & $4(66.7)$ & 0.597 \\
\hline High $(T 1-T 4 a)(n=35)$ & - & - & $7(20.0)$ & $28(80.0)$ & - \\
\hline \multicolumn{6}{|l|}{ Tumor diameter } \\
\hline$>4 \mathrm{~cm}(\mathrm{n}=16)$ & - & - & $5(31.3)$ & $11(68.8)$ & 0.276 \\
\hline$\leq 4 \mathrm{~cm}(\mathrm{n}=25)$ & - & - & $4(16.0)$ & $21(84.0)$ & - \\
\hline \multicolumn{6}{|l|}{ AJCC cancer staging } \\
\hline Tis $(n=12)$ & - & - & $1(8.3)$ & $11(91.7)$ & 0.038 \\
\hline $1-2(n=11)$ & - & - & $1(9.1)$ & $10(90.9)$ & - \\
\hline \multirow[t]{2}{*}{$3-4(n=18)$} & - & - & 7 (38.9) & $11(61.1)$ & - \\
\hline & - & - & - & - & - \\
\hline
\end{tabular}

WHO, World Health Organization; TNM, tumor node metastasis; AJCC, the 7th American Joint Committee on Cancer; -, Negative.

tissues, including 31 (75.6\%) UCB and 13 of 34 (38.2\%) normal bladder tissues, demonstrated no staining. Tumor staining of c-Fos expression was observed in the nucleus staining pattern (Fig. 1).

CD147 immunoreaction positive $(+,++,+++)$ was observed in the 22 cases $(53.7 \%)$ and most tumor cells with diffuse plasma membranous staining of CD147, while none of normal urothelial cells expressed CD147 (Fig. 1), which indicated that the CD147 was more common high expression in human UCB than that in para-cancer normal tissues ( $\mathrm{P}<0.001$; Fig. 1).

A weak inverse correlation was identified (rho=-0.310, $\mathrm{P}=0.049$; Table II) existed in $41 \mathrm{UCB}$ patients between c-Fos 
Table II. CD147, c-Jun and c-Fos expression correlation in 41 patients with urothelial carcinoma of the bladder.

\begin{tabular}{|c|c|c|c|c|c|c|}
\hline \multirow[b]{2}{*}{ Marker } & \multicolumn{2}{|c|}{ CD147 } & \multicolumn{2}{|c|}{ c-Jun } & \multicolumn{2}{|c|}{ c-Fos } \\
\hline & rho & P-value & rho & P-value & rho & P-value \\
\hline CD147 & - & - & 0.024 & 0.881 & -0.310 & 0.049 \\
\hline c-Jun & 0.024 & 0.881 & - & - & 0.233 & 0.142 \\
\hline c-Fos & -0.310 & 0.049 & 0.233 & 0.142 & - & - \\
\hline
\end{tabular}

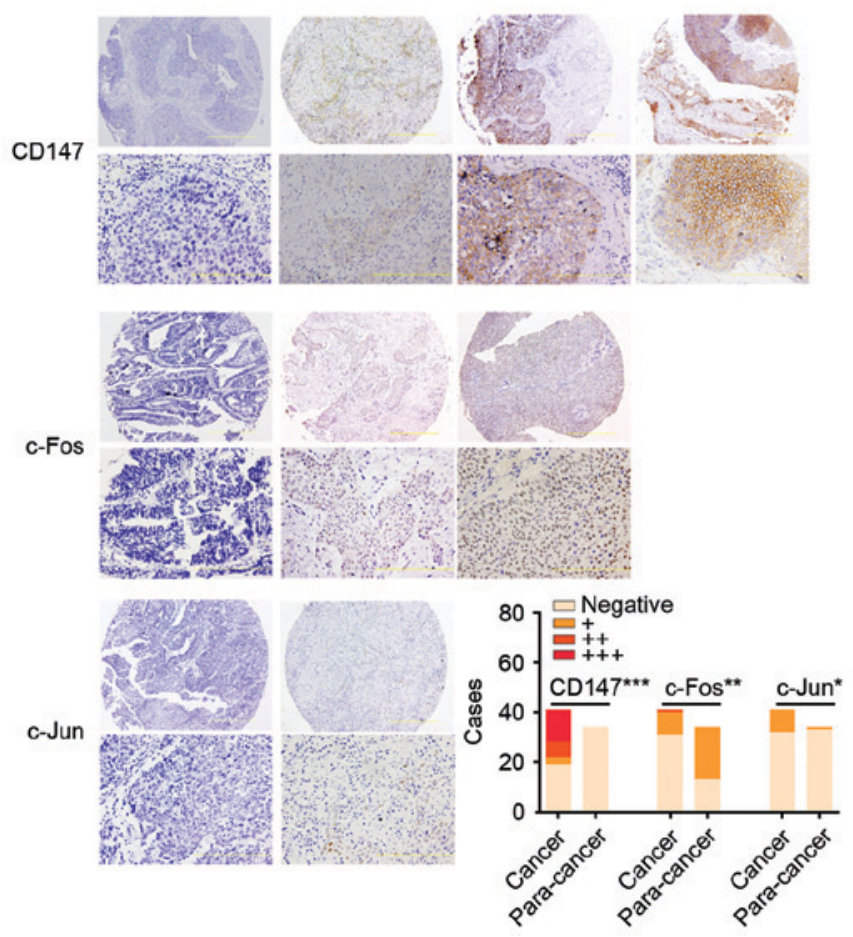

Figure 1. The immunohistochemical staining of CD147, c-Fos and c-Jun in UCB. The expression of c-Jun, c-Fos and CD147 was scored as negative, absence; + , weak; ++ , moderate; and +++ , strong. The images were $\times 100$ (upper row) and $\mathrm{x} 400$ magnification (bottom row). The histogram presents the number of cases with CD147, c-Fos and c-Jun expression in UCB tissues and para-cancer normal tissues. The distribution of 41 cases of UCB and 34 cases of para-cancer tissues is presented in the bar graph. CD147 (negative, $19 ;+, 3 ;++, 6$; and,+++ 13 in UCB. negative, 34 in para-cancer), c-Fos (negative, $31 ;+, 9$; and,++ 1 in UCB. negative, 13; and,+ 21 in para-cancer) and c-Jun (negative, $32 ;+, 9$; in UCB. Negative, 33 ; and,+ 1 in para-cancer), respectively. ${ }^{* * *} \mathrm{P}<0.001,{ }^{* *} \mathrm{P}<0.01$ and ${ }^{*} \mathrm{P}<0.05$ vs. para-cancer. $\mathrm{UCB}$, urothelial carcinoma of the bladder.

expression (negative,,+++ ) and CD147 expression (negative, ,,++++++ ). However, neither $\mathrm{c}-$ Fos expression (rho $=0.233$, $\mathrm{P}=0.142$ ) nor $\mathrm{CD} 147$ (rho $=0.024, \mathrm{P}=0.881$ ) expression had significant association with c-Jun expression (negative, + ).

Clinicopathological parameter relevance and survival analysis. The protein expression of c-Jun was significantly associated with AJCC cancer staging $(\mathrm{P}=0.038)$, while other clinicopathological factors, including age, sex, growth pattern, WHO grade, lymph nodes invasion, tumor node metastatis (TNM) stage and tumor size, had no apparent relationship with c-Jun, c-Fos or CD147 expression (Table I). Neither CD147-c-Jun co-expression (5 cases in 41 UCB patients, 12.2\%), CD147-c-Fos co-expression (2 in $41 \mathrm{UCB}$ patients, 5.0\%), nor c-Fos-c-Jun co-expression (4 in $41 \mathrm{UCB}$ patients, $9.8 \%$ ) were associated with any clinicopathological parameters (Table III).

Univariate survival analyses revealed that high CD147 expression ( $\mathrm{P}=0.038)$, high c-Jun expression $(\mathrm{P}=0.008)$ and high WHO grade $(\mathrm{P}<0.001$; data not shown) were associated with poor OS, whereas high c-Fos expression did not significantly correlate with poor OS for UCB ( $\mathrm{P}=0.225$; Fig. $2 \mathrm{~A}-\mathrm{C})$. Further analysis revealed that either CD147-c-Fos-c-Jun co-expression $(\mathrm{P}=0.004)$ or $\mathrm{CD} 147-\mathrm{c}-J u n$ co-expression $(\mathrm{P}=0.037)$ and c-Fos-c-Jun co-expression $(\mathrm{P}<0.001)$ were also associated with poor OS (Fig. 2D-F). Therefore, it was considered that OS was better in patients who had c-Fos, c-Jun and CD147 all negative expression, or only one of them positive than in those who had double positive or triple positive expression $(\mathrm{P}=0.008$; Fig. 2H).

Multivariate analyses (Table IV) demonstrated that high CD147 expression (hazard ratio $[\mathrm{HR}]=6.889 ; 95 \%$ confidence interval $[\mathrm{CI}]$ : $1.315-36.084 ; \mathrm{P}=0.022)$, high $\mathrm{c}-\mathrm{Fos}$ expression (HR=4.636; 95\% CI:1.128-19.057; $\mathrm{P}=0.033)$, high c-Jun $(\mathrm{HR}=4.589 ; 95 \% \mathrm{CI}: 1.172-17.968 ; \mathrm{P}=0.029)$ and even male sex (HR=0.140; 95\% CI:0.027-0.732; $\mathrm{P}=0.020)$ were independent predictors for poor OS.

\section{Discussion}

The present study demonstrated that increased expression of c-Jun or CD147 proteins was predictive of poor OS for UCB, although c-Fos protein was not significantly associated with OS. Furthermore, both CD147 and c-Jun positive expression, or all positive expression of c-Fos, c-Jun and CD147, served as an index of poor OS for UCB. All above indicated that overexpression of c-Jun and CD147, perhaps including c-Fos may contribute to tumor progression of UCB.

Many genetic factors have been identified as being associated with bladder cancer $(19,20)$. However, not much is understood regarding the molecular mechanisms of its tumorigenesis and tumor progression. CD147 expressed by tumor cells stimulates peritumoral fibroblasts to produce matrix metalloproteinases, thus contributing to tumor invasion and metastasis (21). Previous studies have highlighted the pivotal role of CD147 protein in carcinogenesis and tumor progression. CD147 expression in breast carcinomas is associated with risk factors such as poor histological grade, negative hormone status, the mitotic index and tumor size (22). High CD147 immunostaining scores in hepatocellular carcinomas correlate significantly with tumor grading and tumor-node-metastasis stages (22). It is reported that CD147 is involved in a regulatory 
A

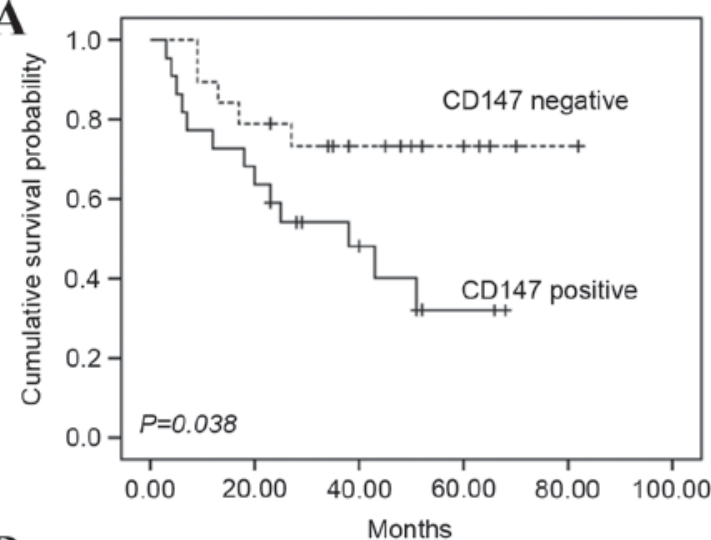

B

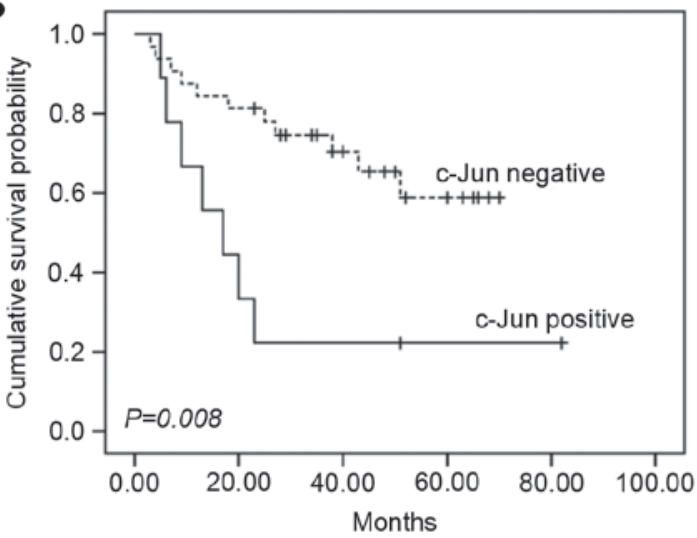

C

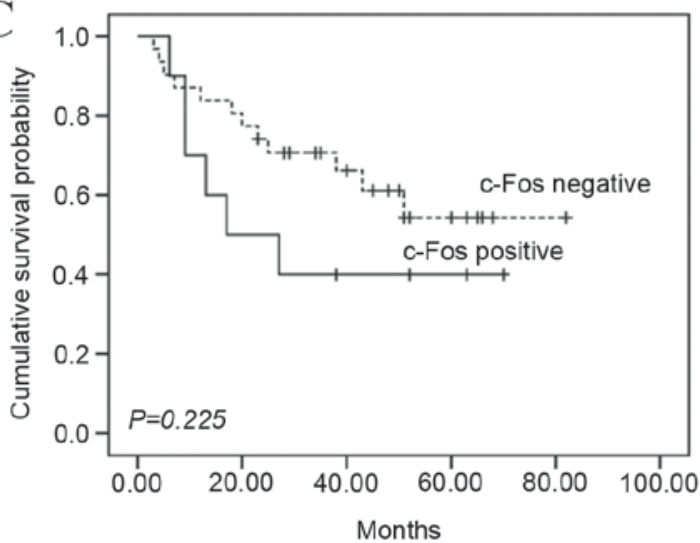

D

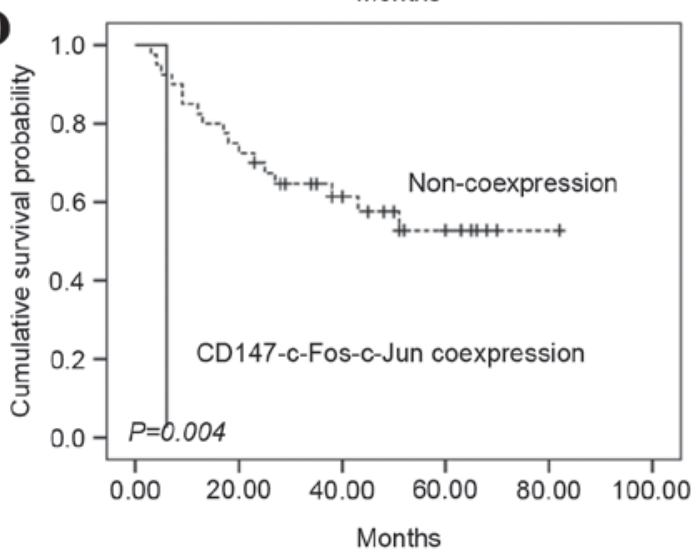

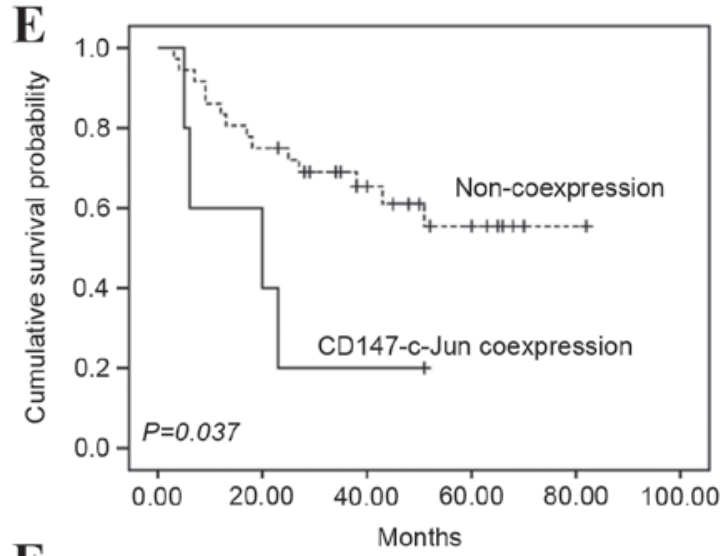
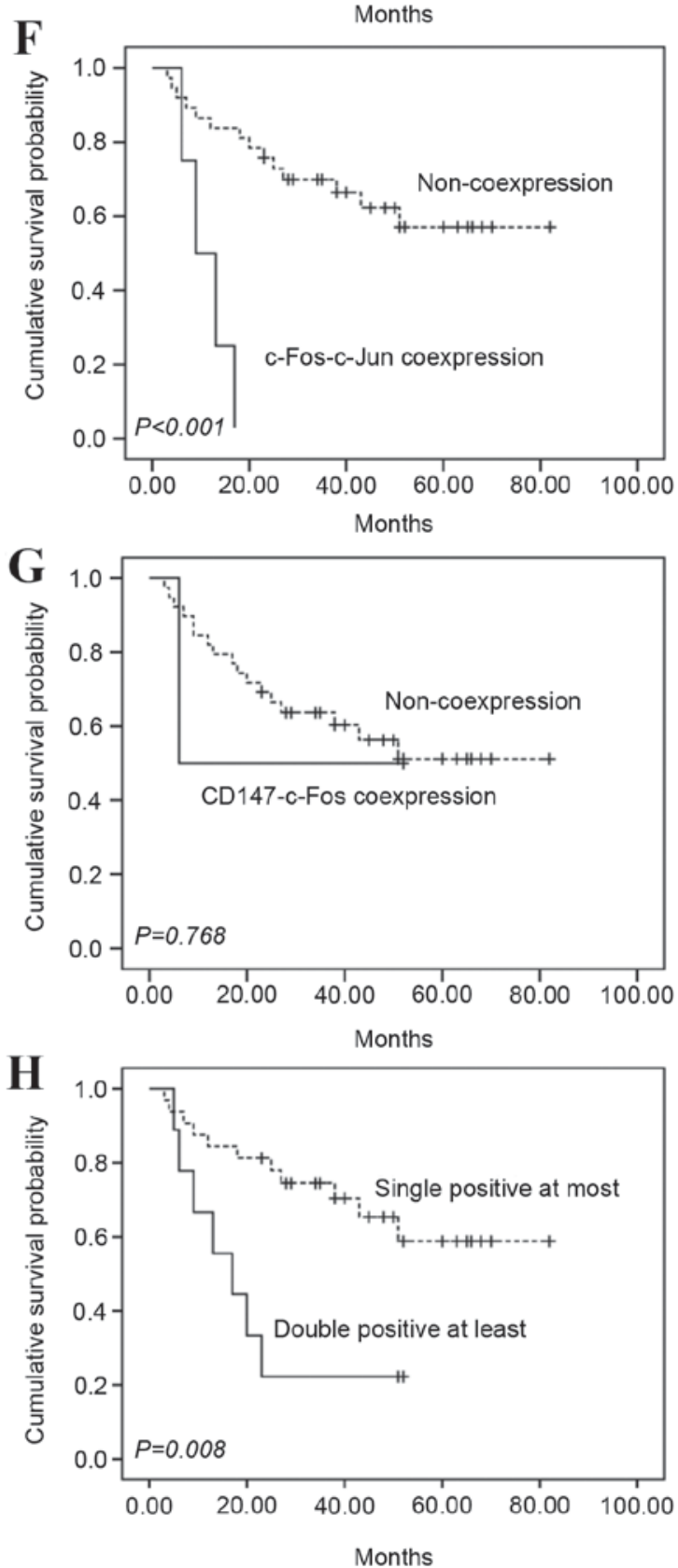

Figure 2. OS analysis of c-Fos, c-Jun and CD147 expression in 41 patients with urothelial carcinoma of the bladder. (A) Patients with CD147 positive expression $(+,++,+++)$ had poorer overall survival rate than those with CD147 negative expression (log-rank test, $\mathrm{P}=0.038)$. (B) Patients with c-Jun positive expression $(+)$ had poorer overall survival rate than those with c-Jun negative expression (log-rank test, $\mathrm{P}=0.008)$. (C) c-Fos positive expression $(+,++)$ did not significantly correlate with poor OS (log-rank test, $\mathrm{P}=0.225$ ). (D) Patients with all the three proteins co-expressed (CD147-c-Fos-c-Jun co-expression) had poorer overall survival rate than the others (log-rank test, $\mathrm{P}=0.004)$. (E) Patients with CD147-c-Jun co-expression had poorer overall survival rate than the others $($ log-rank test, $\mathrm{P}=0.037$ ). (F) Patients with c-Fos-c-Jun co-expression had poorer overall survival rate than the others (log-rank test, $\mathrm{P}<0.001)$. (G) $\mathrm{CD} 147-\mathrm{c}-\mathrm{Fos}$ co-expression did not significantly correlate with poor OS (log-rank test, $\mathrm{P}=0.768)$. (H) OS was better in patients who had c-Fos, c-Jun and CD147 all negative, or single-protein positive expression than in those who had double-protein positive or triple-protein positive expression (log-rank test, $\mathrm{P}=0.008)$. OS, overall survival. 
Table III. CD147, c-Fos, and c-Jun co-expressions in 41 patients with urothelial carcinoma of the bladder.

\begin{tabular}{|c|c|c|c|}
\hline \multirow[b]{2}{*}{ Factor } & \multicolumn{3}{|c|}{ CD147-c-Jun co-expression } \\
\hline & $\begin{array}{l}\text { Co-expression } \\
\quad(\mathrm{n}=5)(\%)\end{array}$ & $\begin{array}{l}\text { Non-co-expression } \\
\qquad(\mathrm{n}=36)(\%)\end{array}$ & P-value \\
\hline \multicolumn{4}{|l|}{ Age (y) } \\
\hline$>71(n=20)$ & $2(10.0)$ & $18(90.0)$ & \multirow[t]{2}{*}{1.000} \\
\hline$\leq 71(n=21)$ & $3(14.3)$ & $18(85.7)$ & \\
\hline \multicolumn{4}{|l|}{ Sex } \\
\hline Male $(n=35)$ & $5(14.3)$ & $30(85.7)$ & \multirow[t]{2}{*}{1.000} \\
\hline Female $(n=6)$ & $0(0)$ & $6(100)$ & \\
\hline \multicolumn{4}{|l|}{ Growth pattern } \\
\hline Papillary $(\mathrm{n}=11)$ & $1(9.1)$ & $10(90.9)$ & \multirow[t]{2}{*}{1.000} \\
\hline Nonpapillary $(\mathrm{n}=30)$ & $4(13.3)$ & $26(86.7)$ & \\
\hline \multicolumn{4}{|l|}{ WHO grade } \\
\hline Low $(n=3)$ & $0(0)$ & $5(100)$ & \multirow[t]{2}{*}{1.000} \\
\hline High $(n=38)$ & $5(13.2)$ & $33(86.8)$ & \\
\hline \multicolumn{4}{|l|}{ Lymph nodes invasion } \\
\hline Negative $(n=37)$ & $5(13.5)$ & $32(86.5)$ & \multirow[t]{2}{*}{1.000} \\
\hline Positive $(n=4)$ & $0(0)$ & $4(100)$ & \\
\hline \multicolumn{4}{|l|}{ TNM stage } \\
\hline Low (Tis) $(\mathrm{n}=6)$ & $0(0)$ & $6(100)$ & \multirow[t]{2}{*}{1.000} \\
\hline High (T1-T4a) (n=35) & $5(14.3)$ & $30(85.7)$ & \\
\hline \multicolumn{4}{|l|}{ Tumor diameter } \\
\hline$>4 \mathrm{~cm}(\mathrm{n}=16)$ & $0(0)$ & $16(100)$ & \multirow[t]{2}{*}{0.137} \\
\hline$\leq 4 \mathrm{~cm}(\mathrm{n}=25)$ & $5(20.0)$ & $20(80.0)$ & \\
\hline \multicolumn{4}{|l|}{ AJCC cancer staging } \\
\hline Tis $(n=12)$ & $0(0)$ & $12(100)$ & \multirow[t]{3}{*}{0.068} \\
\hline $1-2(n=11)$ & $1(9.1)$ & $10(90.9)$ & \\
\hline $3-4(n=18)$ & $4(22.2)$ & $14(77.8)$ & \\
\hline
\end{tabular}

\begin{tabular}{|c|c|c|c|}
\hline \multirow[b]{2}{*}{ Factor } & \multicolumn{3}{|c|}{ CD147-c-Fos co-expression } \\
\hline & $\begin{array}{l}\text { Co-expression } \\
(\mathrm{n}=2)(\%)\end{array}$ & $\begin{array}{l}\text { Non-co-expression } \\
\qquad(\mathrm{n}=39)(\%)\end{array}$ & P-value \\
\hline \multicolumn{4}{|l|}{ Age (y) } \\
\hline$>71(\mathrm{n}=20)$ & $2(10.0)$ & $18(90.0)$ & 0.232 \\
\hline$\leq 71(\mathrm{n}=21)$ & $0(0)$ & $21(100)$ & \\
\hline \multicolumn{4}{|l|}{ Sex } \\
\hline Male $(n=35)$ & $2(5.7)$ & $33(94.3)$ & 1.000 \\
\hline Female $(n=6)$ & $0(0)$ & $6(100)$ & \\
\hline \multicolumn{4}{|l|}{ Growth pattern } \\
\hline Papillary (n=11) & $2(18.2)$ & $9(81.8)$ & 0.067 \\
\hline Nonpapillary $(n=30)$ & $0(0)$ & $30(100)$ & \\
\hline \multicolumn{4}{|l|}{ WHO grade } \\
\hline Low $(n=3)$ & $0(0)$ & $3(100)$ & 1.000 \\
\hline $\operatorname{High}(\mathrm{n}=38)$ & $2(5.3)$ & $36(94.7)$ & \\
\hline \multicolumn{4}{|l|}{ Lymph nodes invasion } \\
\hline Negative $(\mathrm{n}=37)$ & $2(5.4)$ & $35(94.6)$ & 1.000 \\
\hline Positive $(n=4)$ & $0(0)$ & $4(100)$ & \\
\hline \multicolumn{4}{|l|}{ TNM stage } \\
\hline Low (Tis) $(n=6)$ & $0(0)$ & $6(100)$ & 1.000 \\
\hline High $(\mathrm{T} 1-\mathrm{T} 4 \mathrm{a})(\mathrm{n}=35)$ & $2(5.7)$ & $33(94.3)$ & \\
\hline
\end{tabular}


Table III. Continued.

\begin{tabular}{|c|c|c|c|}
\hline \multirow[b]{2}{*}{ Factor } & \multicolumn{3}{|c|}{ CD147-c-Jun co-expression } \\
\hline & $\begin{array}{l}\text { Co-expression } \\
\quad(\mathrm{n}=5)(\%)\end{array}$ & $\begin{array}{l}\text { Non-co-expression } \\
\qquad(\mathrm{n}=36)(\%)\end{array}$ & P-value \\
\hline \multicolumn{4}{|l|}{ Tumor diameter } \\
\hline$>4 \mathrm{~cm}(\mathrm{n}=16)$ & $1(6.2)$ & $15(93.8)$ & 1.000 \\
\hline$\leq 4 \mathrm{~cm}(\mathrm{n}=25)$ & $1(4.0)$ & $24(96.0)$ & \\
\hline \multicolumn{4}{|l|}{ AJCC cancer staging } \\
\hline Tis $(n=12)$ & $0(0)$ & $12(100)$ & 0.548 \\
\hline $1-2(n=11)$ & $1(9.1)$ & $10(90.9)$ & \\
\hline \multirow[t]{2}{*}{$3-4(n=18)$} & $1(5.6)$ & $17(94.4)$ & \\
\hline & \multicolumn{3}{|c|}{ c-Fos-c-Jun co-expression } \\
\hline Factor & $\begin{array}{l}\text { Co-expression } \\
\quad(\mathrm{n}=4)(\%)\end{array}$ & $\begin{array}{l}\text { Non-co-expression } \\
\qquad(\mathrm{n}=37)(\%)\end{array}$ & P-value \\
\hline \multicolumn{4}{|l|}{ Age (y) } \\
\hline$>71(n=20)$ & $1(5.0)$ & $19(95.0)$ & 0.606 \\
\hline$\leq 71(n=21)$ & $3(14.3)$ & $18(85.7)$ & \\
\hline \multicolumn{4}{|l|}{ Sex } \\
\hline Male $(n=35)$ & $3(8.6)$ & $32(91.4)$ & 0.483 \\
\hline Female $(n=6)$ & $1(16.7)$ & $5(83.3)$ & \\
\hline \multicolumn{4}{|l|}{ Growth pattern } \\
\hline Papillary (n=11) & $0(0)$ & $11(100)$ & 0.559 \\
\hline Nonpapillary $(\mathrm{n}=30)$ & $4(13.3)$ & $26(86.7)$ & \\
\hline \multicolumn{4}{|l|}{ WHO grade } \\
\hline Low $(n=3)$ & $0(0)$ & $3(100)$ & 1.000 \\
\hline High $(n=38)$ & $4(10.5)$ & $34(89.5)$ & \\
\hline \multicolumn{4}{|l|}{ Lymph nodes invasion } \\
\hline Negative $(n=37)$ & $4(10.8)$ & $33(89.2)$ & 1.000 \\
\hline Positive $(n=4)$ & $0(0)$ & $4(100)$ & \\
\hline \multicolumn{4}{|l|}{ TNM stage } \\
\hline Low (Tis) $(\mathrm{n}=6)$ & $0(0)$ & $6(100)$ & 1.000 \\
\hline $\operatorname{High}(\mathrm{T} 1-\mathrm{T} 4 \mathrm{a})(\mathrm{n}=35)$ & $4(11.4)$ & $31(88.6)$ & \\
\hline \multicolumn{4}{|l|}{ Tumor diameter } \\
\hline$>4 \mathrm{~cm}(\mathrm{n}=16)$ & $0(0)$ & $16(100)$ & 0.143 \\
\hline$\leq 4 \mathrm{~cm}(\mathrm{n}=25)$ & $4(16.0)$ & $21(84.0)$ & \\
\hline \multicolumn{4}{|l|}{ AJCC cancer staging } \\
\hline Tis $(n=12)$ & $1(8.3)$ & $11(91.7)$ & 0.383 \\
\hline $1-2(n=11)$ & $0(0)$ & $11(100)$ & \\
\hline $3-4(n=18)$ & $3(16.7)$ & $15(83.3)$ & \\
\hline
\end{tabular}

WHO, World Health Organization; TNM, tumor node metastasis; AJCC, the 7th American Joint Committee on Cancer.

loop of miRNA and transcription factors in breast cancer invasion and metastasis (23). In gastric carcinoma, CD147 expression is positively correlated with tumor size, depth of invasion and lymphatic invasion, but not with lymph node metastasis, staging, or differentiation (24). Previous studies of the authors have demonstrated that CD147 may be involved in the progression of prostate cancer and renal cell carcinoma, and can be used as an independent prognostic factor of these cancers $(21,25)$. However, CD147 protein expression patterns within esophageal squamous cell carcinoma and dysplastic lesions are not associated with any of these clinicopathologic factors (26). In the present study, the results indicated that CD147 was overexpressed in human bladder cancer. However, intense expression of CD147 in bladder cancer was 
Table IV. Cox multivariate prognostic analysis for 41 patients with urothelial carcinoma of the bladder.

$95 \%$ CI for HR

\begin{tabular}{|c|c|c|c|c|c|}
\hline \multirow[b]{2}{*}{ Marker } & \multirow[b]{2}{*}{ Risk factor } & \multirow[b]{2}{*}{ P-value } & \multirow[b]{2}{*}{ HR } & \\
\hline & & & & Lower & Upper \\
\hline Sex & Male & 0.020 & 0.140 & 0.027 & 0.732 \\
\hline CD147 & Positive & 0.022 & 6.889 & 1.315 & 36.084 \\
\hline c-Fos & Positive & 0.033 & 4.636 & 1.128 & 19.057 \\
\hline c-Jun & Positive & 0.029 & 4.589 & 1.172 & 17.968 \\
\hline
\end{tabular}

CI, confidence interval; HR, hazard ratio.

not significantly correlated with the TNM stage, WHO grade and tumor size (Table I), which disaccorded with the results of Riethdorf et al (27). In addition, Riethdorf's study identified that the expression levels of CD147 in invasive transitional cell carcinomas of bladder were higher than those in noninvasive tumors (27). These discrepancies suggest that there are different clinical features of CD147 expression in bladder cancer cells of different patients of UCB, in consideration of all cases in the current study with no recurrence and metastasis following transurethral resection and partial or radical cystectomy therapy. It has already been demonstrated that CD147 overexpression is associated with poorer outcome and shorter survival time in patients with solid tumors $(28,29)$. The present findings supported this hypothesis, as CD147 expression was associated with poor survival in univariate analysis (Fig. 2). Cumulatively, these results indicated that CD147 may be one of the key molecular markers to identify high risk of progression in bladder cancer, but other key molecules involved in tumor progression such as transcription factors must be identified to develop more effective therapeutic targets and to supply more reliable judgments of the prognosis of UCB patients. The study of Hagemann et al (30) demonstrated that CD147 activates multiple transcription factors, including AP-1 (c-Fos, c-Jun and FosB) and NF- $\kappa$ B in cardiomyocytes. The AP-1 complex consists of two elements, c-Fos and c-Jun (31). The AP-1 complex has been implicated in the transformation and progression of cancer (32). Although a few studies have discovered some alternative activities of c-Jun like anti-cancer property (33), most research suggests that c-Jun contributes to tumor initiation and increased invasiveness $(18,34)$. In breast cancer, high expression levels of c-Jun in MCF-7 cells can result in an overall increased invasion by increased cellular motility and increased expression of MMP-9 (35). The observed phenotype for MCF-7 cells with c-Jun overexpression is similar to that observed clinically in advanced breast cancer, which had become hormone unresponsive (36). The other most common element of the AP-1 complex, c-Fos, has also been identified as independent predictor of poor survival in breast cancer (14). CD147 has been regarded as a prognostic marker for breast cancer with MMP-9 (37). It seems that there is a reason to examine the hypothesis that c-Jun and CD147, and even c-Fos, are involved in the progression of the human urothelial carcinoma of the bladder, according to the present study results and other previously-mentioned studies. However, future research should aim to elucidate the mechanism of c-Jun, c-Fos and
CD147 in bladder cancer progression by enlarging sample size, and studying in in vitro and in vivo models.

The present study indicated that increased expression of the c-Jun and CD147 proteins, as well as co-expression of CD147-c-Jun, c-Jun-c-Fos, or CD147-c-Jun-c-Fos has prognostic significance for UCB patients. Therefore, high CD147 and c-Jun expression may serve roles in UCB progression and may be diagnostic and therapeutic targets in UCB whether alone or in combination.

\section{Acknowledgements}

This work was supported by grants from National Science and Technology Major Project (grant no. 2013ZX09301301) and National High Technology Research and Development Program (grant no. 2015CB553701).

\section{References}

1. Sharma S, Ksheersagar P and Sharma P: Diagnosis and treatment of bladder cancer. Am Fam Physician 80: 717-723, 2009.

2. Siegel RL, Miller KD and Jemal A: Cancer statistics, 2015. CA Cancer J Clin 65: 5-29, 2015.

3. Van Tilborg AA, Bangma CH and Zwarthoff EC: Bladder cancer biomarkers and their role in surveillance and screening. Int $\mathrm{J}$ Urol 16: 23-30, 2009.

4. Giuliani L, Giberti C, Martorana G, Bonamini A, Natta GD and Rovida S: Results of radical cystectomy for primary bladder cancer. Retrospective study of more than 200 cases. Urology 26: 243-248, 1985.

5. Pagano F, Bassi P, Galetti TP, Meneghini A, Milani C, Artibani W and Garbeglio A: Results of contemporary radical cystectomy for invasive bladder cancer: A clinicopathological study with an emphasis on the inadequacy of the tumor, nodes and metastases classification. The J Urol 145: 45-50, 1991.

6. Xiong L, Edwards CK III and Zhou L: The biological function and clinical utilization of CD147 in human diseases: A review of the current scientific literature. Int J Mol Sci 15: 17411-17441, 2014

7. Zhu S, Li Y, Zhang Y, Wang X, Gong L, Han X, Yao L, Lan M and Zhang W: Expression and clinical implications of HAb18G/CD147 in hepatocellular carcinoma. Hepatol Res 45: 97-106, 2015.

8. Xu J, Xu HY, Zhang Q, Song F, Jiang JL, Yang XM, Mi L, Wen N, Tian R, Wang L, et al: HAb18G/CD147 functions in invasion and metastasis of hepatocellular carcinoma. Mol Cancer Res 5: 605-614, 2007.

9. Xu XY, Lin N, Li YM, Zhi C and Shen H: Expression of HAb18G/CD147 and its localization correlate with the progression and poor prognosis of non-small cell lung cancer. Pathol Res Pract 209: 345-352, 2013.

10. Bhagirath D, Abrol N, Khan R, Sharma M, Seth A and Sharma A: Expression of CD147, BIGH3 and Stathmin and their potential role as diagnostic marker in patients with urothelial carcinoma of the bladder. Clin Chim Acta 413: 1641-1646, 2012. 
11. Bian H, Zheng JS, Nan G, Li R, Chen C, Hu CX, Zhang Y, Sun B, Wang XL, Cui SC, et al: Randomized trial of [131I] metuximab in treatment of hepatocellular carcinoma after percutaneous radiofrequency ablation. J Natl Cancer Inst 106: 239, 2014

12. Hess J, Angel P and Schorpp-Kistner M: AP-1 subunits: Quarrel and harmony among siblings. J Cell Sci 117: 5965-5973, 2004

13. Ye N, Ding Y, Wild C, Shen Q and Zhou J: Small molecule inhibitors targeting activator protein 1 (AP-1). J Med Chem 57: 6930-6948, 2014.

14. Mahner S, Baasch C, Schwarz J, Hein S, Wölber L, Jänicke F and Milde-Langosch $\mathrm{K}$ : C-Fos expression is a molecular predictor of progression and survival in epithelial ovarian carcinoma. Br J Cancer 99: 1269-1275, 2008.

15. Bamberger AM, Milde-Langosch K, Rössing E, Goemann C and Löning T: Expression pattern of the AP-1 family in endometrial cancer: Correlations with cell cycle regulators. J Cancer Res Clin Oncology 127: 545-550, 2001.

16. Gamberi G, Benassi MS, Bohling T, Ragazzini P, Molendini L, Sollazzo MR, Pompetti F, Merli M, Magagnoli G, Balladelli A and Picci P: C-myc and c-fos in human osteosarcoma: Prognostic value of mRNA and protein expression. Oncology 55: 556-563, 1998.

17. Szabo E, Riffe ME, Steinberg SM, Birrer MJ and Linnoila RI: Altered cJUN expression: An early event in human lung carcinogenesis. Cancer Res 56: 305-315, 1996.

18. Vleugel MM, Greijer AE, Bos R, van der Wall E and van Diest PJ: c-Jun activation is associated with proliferation and angiogenesis in invasive breast cancer. Human Pathol 37: 668-674, 2006.

19. Gromova I, Gromov P and Celis JE: bc10: A novel human bladder cancer-associated protein with a conserved genomic structure downregulated in invasive cancer. Int J Cancer 98: 539-546, 2002.

20. Moreira JM, Gromov P and Celis JE: Expression of the tumor suppressor protein 14-3-3 sigma is down-regulated in invasive transitional cell carcinomas of the urinary bladder undergoing epithelial-to-mesenchymal transition. Mol Cell Proteomics 3: 410-419, 2004

21. Han ZD, He HC, Bi XC, Qin WJ, Dai QS, Zou J, Ye YK, Liang YX Zeng GH, Zhu G, et al: Expression and clinical significance of CD147 in genitourinary carcinomas. J Surg Res 160: 260-267, 2010.

22. Zhong WD, Chen QB, Ye YK, Han ZD, Bi XC, Dai QS, Liang YX, Zeng GH, Wang YS, Zhu G, et al: Extracellular matrix metalloproteinase inducer expression has an impact on survival in human bladder cancer. Cancer Epidemiol 34: 478-482, 2010

23. Kong LM, Liao CG, Zhang Y, Xu J, Li Y, Huang W, Zhang Y, Bian $\mathrm{H}$ and Chen $\mathrm{ZN}$ : A regulatory loop involving miR-22, Sp1, and c-Myc modulates CD147 expression in breast cancer invasion and metastasis. Cancer Res 74: 3764-3778, 2014.

24. Zheng HC, Takahashi H, Murai Y, Cui ZG, Nomoto K, Miwa S, Tsuneyama K and Takano Y: Upregulated EMMPRIN/CD147 might contribute to growth and angiogenesis of gastric carcinoma: A good marker for local invasion and prognosis. Br J Cancer 95 $1371-1378,2006$
25. Liang YX, He HC, Han ZD, Bi XC, Dai QS, Ye YK, Qin WJ, Zeng GH, Zhu G, Xu CL and Zhong WD: CD147 and VEGF expression in advanced renal cell carcinoma and their prognostic value. Cancer Invest 27: 788-793, 2009.

26. Ishibashi Y, Matsumoto T, Niwa M, Suzuki Y, Omura N, Hanyu N, Nakada K, Yanaga K, Yamada K, Ohkawa K, et al: $\mathrm{CD} 147$ and matrix metalloproteinase-2 protein expression as significant prognostic factors in esophageal squamous cell carcinoma. Cancer 101: 1994-2000, 2004.

27. Riethdorf S, Reimers N, Assmann V, Kornfeld JW, Terracciano L, Sauter G and Pantel K: High incidence of EMMPRIN expression in human tumors. Int J Cancer 119: 1800-1810, 2006.

28. Zhong WD, Han ZD, He HC, Bi XC, Dai QS, Zhu G, Ye YK, Liang YX, Qin WJ, Zhang Z, et al: CD147, MMP-1, MMP-2 and MMP-9 protein expression as significant prognostic factors in human prostate cancer. Oncology 75: 230-236, 2008.

29. Yu W, Liu J, Xiong X, Ai Y and Wang H: Expression of MMP9 and CD147 in invasive squamous cell carcinoma of the uterine cervix and their implication. Pathol Res Pract 205: 709-715, 2009.

30. Hagemann T, Wilson J, Kulbe H, Li NF, Leinster DA, Charles K, Klemm F, Pukrop T, Binder C and Balkwill FR: Macrophages induce invasiveness of epithelial cancer cells via NF-kappa $B$ and JNK. J Immunol 175: 1197-1205, 2005.

31. Bossis G, Malnou CE, Farras R, Andermarcher E, Hipskind R, Rodriguez M, Schmidt D, Muller S, Jariel-Encontre I and Piechaczyk M: Down-regulation of c-Fos/c-Jun AP-1 dimer activity by sumoylation. Mol Cell Biol 25: 6964-6979, 2005.

32. Ramos-Nino ME and Littenberg B: A novel combination: Ranpirnase and rosiglitazone induce a synergistic apoptotic effect by down-regulating Fra- 1 and Survivin in cancer cells. Mol Cancer Ther 7: 1871-1879, 2008.

33. Yang CW, Lee YZ, Hsu HY, Wu CM, Chang HY, Chao YS and Lee SJ: c-Jun-mediated anticancer mechanisms of tylophorine. Carcinogenesis 34: 1304-1314, 2013

34. Eferl R, Ricci R, Kenner L, Zenz R, David JP, Rath M and Wagner EF: Liver tumor development. c-Jun antagonizes the proapoptotic activity of p53. Cell 112: 181-192, 2003.

35. Briggs J, Chamboredon S, Castellazzi M, Kerry JA and Bos TJ: Transcriptional upregulation of SPARC, in response to c-Jun overexpression, contributes to increased motility and invasion of MCF7 breast cancer cells. Oncogene 21: 7077-7091, 2002.

36. Smith LM, Wise SC, Hendricks DT, Sabichi AL, Bos T, Reddy P, Brown PH and Birrer MJ: cJun overexpression in MCF-7 breast cancer cells produces a tumorigenic, invasive and hormone resistant phenotype. Oncogene 18: 6063-6070, 1999.

37. Zhao S, Ma W, Zhang M, Tang D, Shi Q, Xu S, Zhang X, Liu Y, Song Y, Liu L and Zhang Q: High expression of CD147 and MMP-9 is correlated with poor prognosis of triple-negative breast cancer (TNBC) patients. Med Oncol 30: 335, 2013. 